\title{
IDENTIFYING AND QUANTIFYING MINERAL ABUNDANCE THROUGH VSWIR MICROIMAGING SPECTROSCOPY: A COMPARISON TO XRD AND SEM
}

\author{
Ellen K. Leask ${ }^{1}$ and Bethany L. Ehlmann ${ }^{1,2}$ \\ ${ }^{1}$ Division of Geological \& Planetary Sciences, California Institute of Technology, Pasadena, CA 91125 \\ ${ }^{2}$ Jet Propulsion Laboratory, California Institute of Technology, Pasadena, CA, 91109
}

\begin{abstract}
Visible-shortwave infrared microimaging reflectance spectroscopy is a new technique to identify minerals, quantify abundances, and assess textural relationships at sub-millimetre scale without destructive sample preparation. Here we used a prototype instrument to image serpentinized igneous rocks and carbonate-rich travertine deposits to evaluate performance, relative to traditional techniques: XRD (mineralogical analysis of bulk powders with no texture preservation) and SEM/EDS (analysis of phases and textures using chemical data from polished thin sections). VSWIR microimaging spectroscopy is ideal for identifying spatially coherent rare phases, below XRD detection limits. The progress of alteration can also be inferred from spectral parameters and may correspond to phases that are amorphous in XRD. However, VSWIR microimaging spectroscopy can sometimes be challenging with analyses of very dark materials (reflectance $<0.05$ ) and mineral mixtures occurring at a spatial scales multiple factors below the pixel size. Abundances derived from linear unmixing typically agree with those from XRD and EDS within $~ 10 \%$.
\end{abstract}

Index Terms - Imaging spectroscopy, quantitative mineralogy, Mars analogue, petrology, planetary instruments

\section{INTRODUCTION}

Visible to shortwave infrared (VSWIR) reflectance spectroscopy has been used for large-scale compositional mapping on Earth, Mars, and other planets at scales of tens of metres to km per pixel [e.g., 1-4]. The prototype UltraCompact Imaging Spectrometer (UCIS) [5], implemented for this work in microscopic mode, allows direct comparison with remote sensing data and identification of minerals and textures at microscale. VSWIR microimaging spectroscopy is new geosciences technique for performing mineralogic analysis, which is non-destructive, preserves petrographic relationships, and requires minimal sample preparation [6]. Because each mineral has a different set of conditions under which it is stable, identifying multiple minerals while preserving textural context allows for tighter constraints on geochemical conditions at the time of formation. It is not currently feasible to make and study thin sections on rocks in situ on planetary surfaces; VSWIR microimaging spectroscopy provides an alternative method to obtain a similar level of petrographic information.

Samples from the Samail Ophiolite (Oman) are used as an analogue for Martian carbonate and serpentine deposits [e.g., 7] to evaluate the ability of VSWIR microimaging spectroscopy to identify minerals present, distinguish carbonates of different chemistries from minerals with absorptions at similar wavelength positions, and to quantify mineral abundances. This work is part of a larger project to establish methods of identifying and quantifying carbonate content at multiple spatial scales using reflectance spectroscopy. Here, we compare microimaging spectroscopy results to current standard geosciences techniques for mineralogy and petrology: powder XRD for bulk mineralogy and SEM/EDS for texture and phase chemistry.

\section{METHODS}

\subsection{Data Collection}

Fifteen cut rock samples from the Samail Ophiolite (Oman), representing a suite of textures and compositions, were measured with UCIS over the wavelength range 0.5-2.5 $\mu \mathrm{m}$, with $10 \mathrm{~nm}$ spectral resolution, and a pixel footprint of 81 x81 $\mu \mathrm{m}$ (e.g., Fig. 1). Each sample takes $\sim 3$ minutes to image. Two or more subsamples of each rock were sent to two external laboratories (ActLabs, K-T Geoservices) for xray diffraction (XRD) analyses of powdered samples for mineral identification and quantitative abundance estimates. Two samples were polished, imaged with UCIS, then carbon coated, and imaged on a scanning electron microscope (SEM). Energy-dispersive spectroscopy (EDS) maps were obtained over the area of the sample, providing elemental abundance data for direct comparison with UCIS data.

\subsection{Endmember Selection}

Principal component analyses (PCA) using built-in algorithms in ENVI were run on each image. The first $\sim 20$ principal components were investigated through ENVI's ndimensional visualizer; potential endmembers were selected based on the vertices of the point cloud. A parsimonious set 
of endmembers was determined for each sample, based on the potential endmembers and expert user analysis. Regions of interest ( 15 pixels) were drawn for each endmember, taking the mean spectrum for the region. Automated endmember selection algorithms (K-means, ISODATA) were tested, but failed to identify minerals of interest (strongly biased to overall reflectance and not sensitive to subtle differences in shape or band position).

\subsection{Linear Unmixing}

At this spatial scale, many pixels consist of a single mineral phase. As a first-order approximation, we use linear spectral unmixing to estimate the abundance of phases, assuming most mixed spectra result from areal 'checkerboard' mixtures, where endmember spectra combine linearly, proportional to their areal abundance within a pixel. Best-fit abundance estimates are obtained using a fully constrained (sum to 1), non-negative linear least squares algorithm [8]. If initial output images were noisy and did not follow observable textures in the rock, endmembers were re-
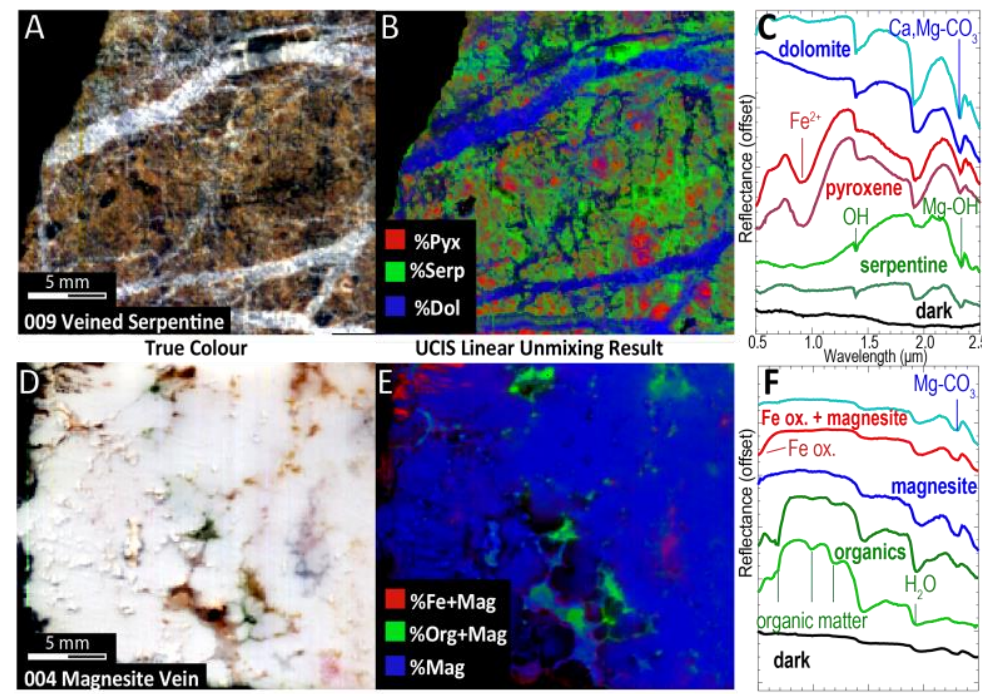

Fig.1. Linear unmixing examples. A-C: Veined serpentinite sample. Unmixing results $(B)$ highlight veins and remnant pyroxene (endmember spectra shown in C). D-F: Magnesite vein sample, with organic matter (green) in porous areas. chosen until the output was coherent. Endmember selection was refined until average RMS errors were under 0.05. Nonlinear algorithms were not considered in this study, as particle size is not consistent and optical constants are not available for natural samples.

\subsection{EDS Comparison}

UCIS images were coregistered with EDS elemental abundance maps for two samples. Single-pixel endmembers corresponding to the same physical locations were used to unmix both the elemental abundance and spectral data independently via the same algorithm. Mineral phases were also mapped via absorption band depth and band centre parameters in VSWIR data, and element ratios and abundances in EDS data, allowing direct spatial correlation of elemental and spectral data on a pixel-by-pixel basis.

\section{RESULTS}

\subsection{Mineral Identification}

In 13 of 15 samples, UCIS identifies all the same major (>5\% abundance) mineral phases as XRD analysis (Fig. 2). Exceptions are a very dark sample (002), where UCIS did not detect 10-19\% olivine, and brighter sample (011), where UCIS did not identify $\sim 6 \%$ quartz found by XRD. Notably, UCIS data reliably differentiated between carbonate minerals (calcite, dolomite, and magnesite) and serpentine with very similar overall spectra, based on the exact position of the $2.3 \mu \mathrm{m}$ absorption $[9,10]$ (Fig. 2C). Specific carbonate minerals can be clearly distinguished when each phase extends over several pixels. Sub-pixel mixtures of carbonate phases (such as dolomite and calcite in the pore spaces of a magnesite vein sample (004, 008), seen with SEM) cannot always be uniquely distinguished. Phases that are present only within fine-grained mixtures may sometimes be identified if they possess distinctive (if subtle) absorptions; for instance, brucite in sample 021 was identified by a hydroxyl absorption at $1.4 \mu \mathrm{m}$, in addition to a slight $2.48 \mu \mathrm{m}$ absorption and sharp $0.95 \mu \mathrm{m}$ band. In magnesite vein samples 004 and 008, absorption features consistent with organic matter are also noted - a sharp 'red edge' at $\sim 0.8 \mu \mathrm{m}$, and minor absorptions in the $1-2 \mu \mathrm{m}$ range associated with C-H, N-H, and C-O bonds [11] (Fig. 1F).

\subsection{Mineral Quantification}

Images from the linear unmixing highlight expected petrologic textures for rocks of this type: the 'dolomite' endmember occurs in veins, while the 'pyroxene' endmember is concentrated in the centres of clasts (Fig. 1B). The algorithm works even for discriminating spectrally similar endmembers; for example, sample 004 has 6 different magnesite-type endmembers, including stripes of magnesite with a reddish (ferric) coating and organic-rich magnesite within porosity (Fig.1E).

For most samples, abundance estimates between UCIS and XRD are the same within $10-15 \%$ (median total difference per sample); scatter between multiple XRD analyses of different subsamples of the same rock is $5-10 \%$. (The two darkest samples, 002 and 016, were not included in this, as less than half the sample can be reliably classified with VSWIR as implemented here, discussed further below). Given the heterogeneous nature of many of the samples, including individual clasts comprising a large portions of some samples (e.g., 001, 018, 021), natural variance in abundance is expected. Furthermore, XRD abundances are reported in wt. \%, while UCIS are areal \%. UCIS data consistently show higher pyroxene and serpentine in samples with a high amorphous component and/or heavy veining $(003,017,018,019,020,021)$, but this may be a true 


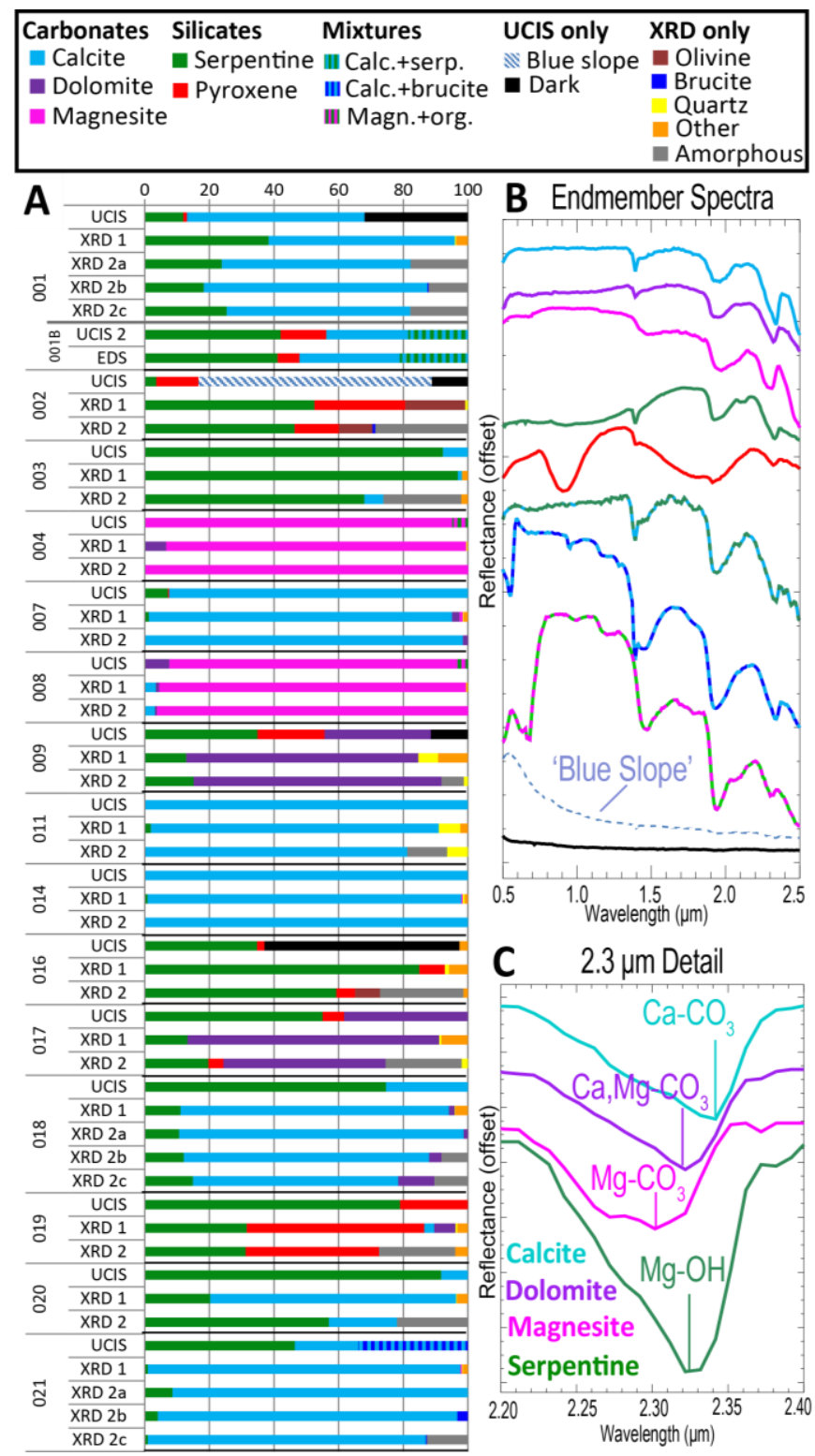

Fig.2. Comparison of linear unmixing results (UCIS) and XRD abundance estimates (A). B: Examples of endmember spectra used in unmixing. C: Zoom showing subtle differences in the $2.3 \mu \mathrm{m}$ absorption between carbonates and serpentine.

difference in sensitivity of the techniques to incipient alteration, as discussed below.

Direct comparison of areal abundances from EDS and UCIS is possible for sample 001 (Fig. 3C, D). Here, determinations are very similar (EDS (UCIS)): 41 (42)\% serpentine, 31 (25)\% calcite, 21 (18)\% mixed carbonate and serpentine, and 7 (14) \% pyroxene.

\section{DISCUSSION}

Overall, this study demonstrates that VSWIR microimaging spectroscopy provides bulk mineralogy estimates of comparable accuracy to XRD for most of this set of samples, and provides mineralogical and textural information comparable to SEM/EDS. Below we highlight some of the key challenges as well as the key advantages of this new technique vs. existing geological methods for mineralogy.

\subsection{Microimaging Spectroscopy Challenges}

\subsubsection{Dark Minerals and 'Blue Slope' Spectra}

There are at least two related processes responsible for the difficulty in identifying minerals in dark $(<0.05$ reflectance $)$ samples: (1) dark phases absorbing most of the photons, leading to little reflected light back to the detector, and (2) internal scattering caused by extremely fine-grained opaque minerals. Issue (1) can be addressed simply by increased acquisition times to average many spectra and optimization of instrument design to improve SNR (this particular instrument configuration, which utilizes a not-full performance detector spare leftover from a flight project, was not optimized). Issue (2) is harder to address - the presence of $<2 \mu \mathrm{m}$ opaques within brighter minerals (Fig. 3F) may cause Rayleigh or Mie type scattering at SWIR wavelengths, creating a 'blue slope' that dominates the spectral signature for that pixel.

\subsubsection{Minerals without strong VSWIR absorptions}

Quartz and other minerals such as feldspars do not have strong VSWIR absorptions, and so they are not expected to be detected with this technique. However, it is sometimes possible to infer their presence by identifying their alteration products-here, we searched unsuccessfully for an absorption at $2.2 \mu \mathrm{m}$ associated with hydrated silica [12].

\subsubsection{Grain Size and Mixed Phases}

Four samples (001, 004, 008, and 021) include a mixedmineral endmember. Mixed mineral endmembers were chosen when either spectral properties of the mixed phase were significantly different from either endmember or where no pure second endmember pixels could be found (e.g. calcite with brucite, magnesite with organics). Fine grained minerals are not expected to combine linearly. Using a mixed endmember in linear spectral unmixing is a way to estimate how much of the mixed phase is present, but we cannot quantify how much each contributes to the mixture. Additionally, linear mixture modelling is controlled by the overall albedo of the phase in question, a parameter controlled by grain size as well as composition. We chose to implement a simple algorithm (endmember selection and linear unmixing) as a fast way to apply VSWIR microspectroscopy. It is clear that as VSWIR microimaging spectroscopy develops as a mineralogic tool, more sophisticated algorithms will be developed and implemented, including nonlinear mixture modelling (e.g. Hapke [13]). Furthermore, there is a need for further tests with samples (e.g. siltstones and mudstones) where grains are mostly below the spatial resolution of the instrument. Instrument design and algorithms can then be optimized for rock samples of multiple grain sizes, including for performance within a few factors of the diffraction limit $(\sim 10 \mu \mathrm{m} /$ pixel for VSWIR). 


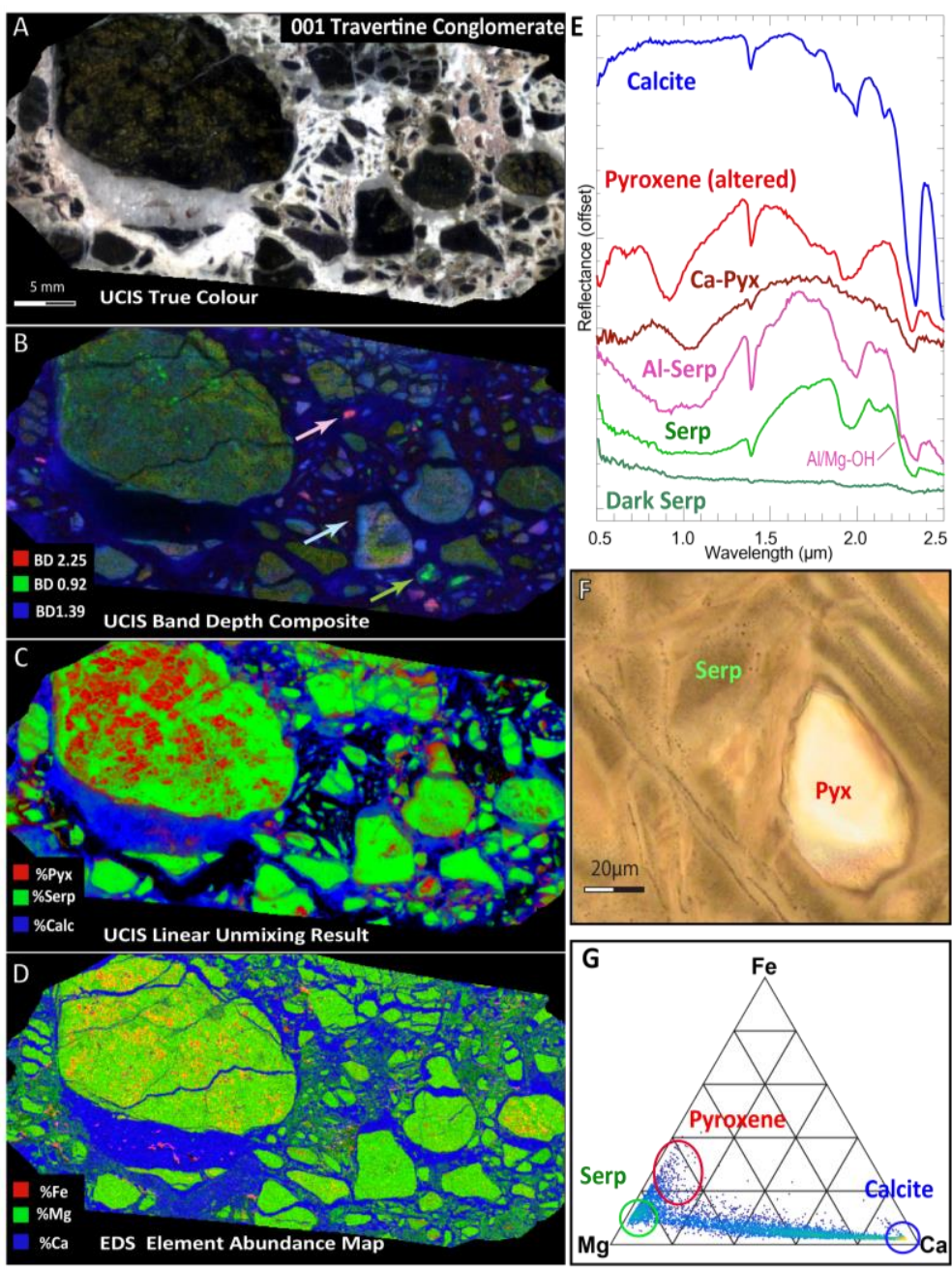

Fig. 3. UCIS and EDS for a travertine conglomerate sample. RGB composite of key band depths (B) highlights rare clasts and textures. Unmixing results in $\mathrm{C}$ (using endmember spectra $(\mathrm{E})$ ) closely correspond to elemental abundances from EDS (D). F) Thin section photomicrograph showing microopaques associated with pyroxene alteration. G) Ternary plot of EDS data showing serpentine, pyroxene, and calcite endmembers with a clear mixing line between serpentine and calcite, with endmembers used in (D).

\subsection{Microimaging Spectroscopy Advantages}

\subsubsection{Rare and Amorphous Phases}

UCIS identifies spatially coherent rare phases, below the detection limit of powder XRD. Distinctive $0.9-\mu \mathrm{m}$ pyroxene absorptions [14; Fig 1C, 2B, 3E] are seen in samples 001, 007, and 009, though pyroxene is not detected by XRD. Petrographic analysis of thin sections confirms the presence of pyroxene. Pixels with pure pyroxene are rare; most show different degrees of partial alteration to serpentine (sharp 1.4- and 2.3- $\mu \mathrm{m}$ absorptions; Fig. 1C, 1F). This may be included in the 'amorphous' fraction of XRD results; hence with UCIS, we can get a clearer picture of the bulk mineral composition in such samples and the processes driving alteration by the ability to simultaneously detect the primary mineral and secondary product. Several clasts in conglomerate sample 001 were identified as Albearing serpentine or chlorite due to a small 2.25$\mu \mathrm{m}$ absorption (caused by $\mathrm{Al} / \mathrm{Mg}-\mathrm{OH}$ vibrational overtones [15]; Fig.3B, E). EDS elemental mapping confirms that these clasts contain much more $\mathrm{Al}$ than other clasts.

\subsubsection{Textural Interpretation}

A key advantage of VSWIR microimaging spectroscopy vs. XRD is its ability to obtain mineralogical information with petrographic context, allowing process interpretation. For example, environmental history can be seen in travertine conglomerate sample 001: (1) most pyroxene is altered to serpentine in a typical ultramafic mesh-like alteration texture (green in Fig. 3B; red in Figs. 3C,D); (2) large-grained calcite veins form (dark blue, Fig. 3C,D), possibly associated with $1.4 \mu \mathrm{m}(\mathrm{OH})$ alteration in adjacent serpentine (light blue, Fig.3B); (3) clasts are broken up and rounded through a sediment transport process; (4) clasts are cemented in a fine-grained calcite/serpentine matrix. EDS also retains spatial relationships, but requires a high degree of sample preparation, which is expensive and time consuming ( $\sim 7$ hours vs $\sim 3$ mins), and is insensitive to $\sim$ isochemical alteration (elemental abundances of serpentine and pyroxene are very similar (Figs. 3C vs. D, 3G)).

\section{CONCLUSIONS}

VSWIR microimaging spectroscopy combined with linear spectral unmixing provides estimates of quantitative mineral abundance consistent with abundance estimates from XRD (weight \%) and EDS (area \%). The UCIS prototype instrument demonstrates the ability to identify all VSWIRactive phases and differentiate between carbonate minerals and other minerals like serpentines with major absorptions in the same wavelength region ( 10 $\mathrm{nm}$ difference in band position), though further instrument and algorithm optimization is needed to deal with $<0.05$ albedo phases and fine-grained mixtures at sub-pixel scale. UCIS is especially well-suited to identify spatially coherent rare phases that would be missed by traditional techniques such as XRD and is an effective, rapid method to survey a set of samples with minimal preparation.

Acknowledgements. Thanks to A. Fraeman, C. Sanders, and B. Van Gorp for assistance with sample data acquisition and to the entire JPL UCIS team. This work was partially supported by a NASA Mars Fundamental Research Program grant (\#NNX12AB42G) to B.L.E., a JPL-RTD/PDF to B.L.E. and D. Blaney, and a private grant to Caltech from the Rose Hills Foundation. 


\section{REFERENCES}

[1] Green, R. O., et al. (1998). "Imaging spectroscopy and the airborne visible/infrared imaging spectrometer (AVIRIS)." Remote Sensing of Environment 65(3): 227248.

[2] Bibring, J.-P., et al. (2005). "Mars surface diversity as revealed by the OMEGA/Mars Express observations." Science 307(5715): 1576-1581.

[3] Murchie, S., et al. (2007). "Compact reconnaissance imaging spectrometer for Mars (CRISM) on Mars reconnaissance orbiter (MRO)." Journal of Geophysical Research: Planets (1991-2012) 112(E5).

[4] DeSanctis, M. C., et al. (2013). "Vesta's mineralogical composition as revealed by the visible and infrared spectrometer on Dawn." Meteoritics \& Planetary Science 48(11): 2166-2184.

[5] Van Gorp, B., et al. (2014). "Ultra-compact imaging spectrometer for remote, in situ, and microscopic planetary mineralogy." Journal of Applied Remote Sensing 8(1): 084988-084988.

[6] Greenberger, R. N., et al. (2015). "Imaging spectroscopy of geological samples and outcrops: Novel insights from microns to meters." GSA Today 25(12).

[7] Ehlmann, B. L. and J. F. Mustard (2012). "An in-situ record of major environmental transitions on early Mars at Northeast Syrtis Major." Geophysical research letters 39(11).

[8] Heylen, R., et al. (2011). "Fully constrained least squares spectral unmixing by simplex projection." Geoscience and Remote Sensing, IEEE Transactions on 49(11): 4112-4122.

[9] Gaffey, S. J. (1987). "Spectral reflectance of carbonate minerals in the visible and near infrared $(0.35-2.55 \mathrm{um})$ : Anhydrous carbonate minerals." Journal of Geophysical Research: Solid Earth (1978-2012) 92(B2): 1429-1440.

[10] Clark, R. N. (1999). "Spectroscopy of rocks and minerals, and principles of spectroscopy." Manual of remote sensing 3: 3-58.

[11] ASD Inc. (2013). "Near-IR Absorption Bands." Retrieved 02-07-16, from http://cdn2.hubspot.net/hub/45853/file-244381196pdf/docs/near-

ir_absorption_bands_chart.pdf?t=1454607097572.
[12] Lauger, K. and O. Florke (1974). "Near infrared absorption spectra $(4,000-9,000 \mathrm{~cm}-1)$ of opals and the role of 'water'in these $\mathrm{SiO} 2 \cdot \mathrm{nH} 2 \mathrm{O}$ minerals." Fortschr. Miner 52: $17-51$.

[13] Hapke, B. (1981). "Bidirectional reflectance spectroscopy: 1. Theory." Journal of Geophysical Research: Solid Earth 86(B4): 3039-3054.

[14] Cloutis, E. A. and M. J. Gaffey (1991). "Pyroxene spectroscopy revisited: Spectral-compositional correlations and relationship to geothermometry." Journal of Geophysical Research: Planets 96(E5): 22809-22826.

[15] Bishop, J., et al. (2008). "Reflectance and emission spectroscopy study of four groups of phyllosilicates: Smectites, kaolinite-serpentines, chlorites and micas." Clay Minerals 43(1): 35-54. 